\title{
Electroencephalographic study of real-time arithmetic task recognition in learning disabilities children
}

\author{
Phakkharawat Sittiprapaporn ${ }^{1,2}$, Shao-Chin Chang ${ }^{3}$ \\ ${ }^{1}$ Assistant Professor, School of Anti-Aging and Regenerative Medicine, Mae Fah Luang University, Bangkok, Thailand, \\ ${ }^{2} \mathrm{Head}$, Brain Science and Engineering Innovation Research Unit, School of Anti-Aging and Regenerative Medicine, \\ Mae Fah Luang University, Bangkok, Thailand, ${ }^{3}$ Ph.D. Program of Electrical and Communications Engineering, Feng \\ Chia University, Taichung, Taiwan
}

Background: Mathematics form is universally understood in modern society. It is natural to ask whether there is neurophysiological evidence for putative cognitive components of the task and the practice in mental arithmetic will lead to a detectable transition in primary locus of brain activity. Aims and Objectives: The purpose of this study was to determine the effect of real-time arithmetic recognition task for cognitive performance and electroencephalographic activities. Materials and Methods: While practicing the real-time arithmetic recognition task named SpeedMath developed by NeuroSky, Inc., electroencephalographic activities were also recorded by using the commercial lightweight electroencephalographic device, Mindwave Mobile, NeuroSky, Inc. Eight participants included learning disabilities children participating in this study. All participants were instructed and trained to practice the simple the arithmetic recognition task which included arithmetic and mathematic skills. Results: The results showed that both alpha and beta frequency bands were increased with statistically significant at the 0.05 level. Conclusions: The real-time arithmetic recognition task might improve the real-time arithmetic recognition task and performance.
Access this article online Website:

http://nepjol.info/index.php/AJMS DOI: 10.3126/ajms.v10i1.21035 E-ISSN: 2091-0576 P-ISSN: 2467-9100

Key words: Brain; Brain activity; Arithmetic; Cognition; Brain training

\section{INTRODUCTION}

Mathematics and arithmetic form of a prototypical cognitive system is almost universally understood in modern society. System or domain knowledge and the skills to use that knowledge are acquired relatively early in life, and are used in grossly similar ways by all adults. ${ }^{1}$ In several previous studies, cognitive psychologists had employed mental arithmetic problems as a paradigm to study higher order mental processes. Some important facts are beginning to emerge from behavioral studies of arithmetic and mathematical processes. Much of this empirical work using a mental arithmetic task are scored primarily according to speed of answer production. ${ }^{1,2}$ However, it is natural to ask whether there is neurophysiological evidence for putative cognitive components of the task and about possible systematic relationships between these brain activities and variables were known to affect speed and accuracy of performance. ${ }^{3}$ Therefore, it is entirely reasonable to expect that practice in mental arithmetic will lead to a detectable transition in primary locus of brain activity. ${ }^{1-3}$

Brain is an organ that is easily affected by the physical changes, especially in the modern time when people are easily poisoned by the increasing pollution. ${ }^{4-6}$ Electroencephalography (EEG) is traditionally used to measure the human brain waves. Each type of brain wave is associated with one's state of consciousness and different mood and emotional states. ${ }^{7,8}$ The electroencephalography thus offers a way to monitor brain activity in real time and to track any transitions that parallel changes in behavior. ${ }^{9}$ 
There are some evidences of an association between slow brain potentials and the task demands of mental arithmetic. ${ }^{9}$ One finding is that more difficult tasks are associated with more positive amplitude levels. ${ }^{10,11}$ Additionally, mental arithmetic tasks seem to be associated with a relative positivity over the frontal cortex. ${ }^{11-15}$ However, the interpretation of this frontal positivity is still somewhat controversial. Rosler and Heil ${ }^{13}$ related the frontal positivity to the mental operation of division and also related Ruchkin's study ${ }^{15}$ to the allocation of resources and/or to the coordination of processing states. ${ }^{13,15}$ In the present study, the measurement of electroencephalographic activity was done by letting participants who were learning disabilities, did the realtime arithmetic recognition task operated by SpeedMath program which was developed by NeuroSky, Inc., and then measured the electroencephalographic activity during the real-time arithmetic performing.

\section{MATERIALS AND METHODS}

Participants: The experiment was done with eight learning disabilities children, aged between 5-12 years old, who were in good health, no record of brain surgery, not taking medicines or drugs that affect to nervous system. Exclusion criteria included left handedness; history of medical and neurologic diseases; psychiatric disorders; head trauma; assumption of central nervous system active drugs in the two weeks prior to study entry; and presence of electroencephalographic abnormalities at the baseline electroencephalographic recording. After receiving information about the aims of the study, we provided written consent to parents to sign according to the Helsinki declaration standards and was approved by the local institutional review board of Mae Fah Luang University, Thailand.

Tools and Equipment: Recording personal information including age, gender, nationality, and history of illness were recorded. The electroencephalography was applied both before and after brain training. The neuro headset was used to record, analyze, and display the output of electroencephalographic activities. In this study, the effectiveness of commercially available lightweight electroencephalographic devices, NeuroSky's Mindwave Mobile (NeuroSky, Inc.), was considered. Via the application of a single electrode and signal-processing unit in a head band arrangement, the Mindwave Mobile provides two 100-state outputs operating at $1 \mathrm{~Hz}$. Although the Mindwave Mobile provided a much coarser picture of brain activity than multi-electrodes electroencephalographic or other technologies, the principle advantage of the Mindwave Mobile was its unobtrusive nature, which minimizes the aforementioned difficulties in conducting accurate user studies. In this study, the principles of analytical check were based on the International 10-20 system by displaying the electroencephalographic output. The electroencephalographic frequency analysis was performed by means of a Fast Fourier Transform (FFT) algorithm, with a 2-second interval on the electroencephalographic signal. A single-channel electroencephalogram was designed to collect brain activity data from the participants' brain. Simplicity and efficiency were the main reasons for designing a single-channel electroencephalographic monitoring system. The electroencephalographic data was analyzed offline and the following frequency bands were computed: delta ranging from 0.1 to $3.0 \mathrm{~Hz}$, theta (4.0 to $7.0 \mathrm{~Hz})$, alpha (8.0 to $13.0 \mathrm{~Hz})$, beta $(14.0$ to $30.0 \mathrm{~Hz})$, and gamma (31.0 to $47.0 \mathrm{~Hz}$ ), respectively.

SpeedMath, developed by NeuroSky, Inc., used in this study is a computer-based task which is designed to train participants' arithmetic skills to be more precise and efficient. After participants had completed a problem set, the changing of attention levels was then reviewed. In the SpeedMath post reports, each vertical line in the post report represents an answered problem. It helps to build up participants' quick-thinking math skills by answering problems both accurately and quickly. Actually, the SpeedMath is the game for everyone. This game lets participants build up their math skills while working on their problem areas. As the participants improve, so will their attention level and their problem-solving skills. Measuring electroencephalographic activity while real-time arithmetic recognition task was also compared to baseline.

Statistical Analysis: Analyzed the basic demographic data of participants by using descriptive analysis with qualitative data summarized in term of frequency and percentage, with quantitative data summarized in term of average and standard deviation. Using inferential statistic compares each type of electroencephalographic activity by compared $t$-test. Every test was set to the statistic significant at $p<0.05$.

\section{RESULTS}

According to Table 1, it was found that after brain training, alpha and beta frequency bands gradually increased while delta, theta, and gamma frequency bands did not change. In addition, both alpha and beta frequency bands increased with statistically significant at the 0.05 level while performing the real-time arithmetic recognition task.

The delta frequency band did not change while performing the real-time arithmetic recognition task compared to baseline (baseline: $0.63 \pm 0.012 \mu \mathrm{V}$; arithmetic task: 


\begin{tabular}{|c|c|c|c|c|c|}
\hline \multirow[t]{2}{*}{ Frequency bands } & \multicolumn{2}{|c|}{ Before brain training } & \multicolumn{2}{|c|}{ After brain training } & \multirow[t]{2}{*}{$P$-value } \\
\hline & Mean & SD & Mean & SD & \\
\hline Delta & 0.63 & 0.012 & 0.74 & 0.15 & 0.24 \\
\hline Theta & 0.21 & 0.017 & 0.41 & 0.21 & 0.95 \\
\hline Alpha & 0.37 & 0.025 & 0.71 & 0.36 & $0.01^{*}$ \\
\hline Beta & 0.03 & 0.013 & 0.74 & 0.23 & $0.03^{*}$ \\
\hline Gamma & 0.48 & 0.83 & 0.36 & 0.09 & 0.07 \\
\hline
\end{tabular}

$0.74 \pm 0.15 \mu \mathrm{V} ; \mathrm{t}(15)=1.57 ; p=0.24)$ whereas the theta frequency band increased with no statistically significant while performing the real-time arithmetic recognition task compared to baseline (baseline: $0.21 \pm 0.017 \mu \mathrm{V}$; arithmetic task: $0.61 \pm 0.21 \mu \mathrm{V} ; \mathrm{t}(15)=1.81 ; p=0.95)$. On the other hand, it was found that alpha frequency band was found to be increased with statistically significant at the 0.05 level while performing the real-time arithmetic recognition task compared to baseline (baseline: $0.37 \pm 0.025 \mu \mathrm{V}$; arithmetic task: $0.71 \pm 0.36 \mu \mathrm{V} ; \mathrm{t}(15)=0.41 ; p=0.01)$ while the beta frequency band also increased with statistically significant at the 0.05 level while performing the realtime arithmetic recognition task compared to baseline (baseline: $0.03 \pm 0.013 \mu \mathrm{V}$; arithmetic task: $0.74 \pm 0.23 \mu \mathrm{V}$; $\mathrm{t}(15)=0.13 ; p=0.03)$. Finally, it was found that gamma frequency band did not change while performing the realtime arithmetic recognition task compared to baseline (baseline: $0.48 \pm 0.83 \mu \mathrm{V}$; arithmetic task: $0.36 \pm 0.09 \mu \mathrm{V}$; $\mathrm{t}(15)=1.04 ; p=0.07$.

\section{DISCUSSION}

Our findings revealed that after brain training, alpha and beta frequency bands were increased with statistically significant while performing the real-time arithmetic recognition task. Participants have shown significantly mean improvement in speed, memory, attention, flexibility and problem solving, respectively. This is consistent with findings in several previous studies including in healthy adults with mild cognitive impairment and in chemotherapy-treated cancer survivors, respectively. ${ }^{16-18}$

Neuropsychological studies indicated that the frontal brain is involved whenever mental activity is deliberate and controlled. 16,19,20 The more the processing of the arithmetic task becomes automatized, the less frontal cortical activity will be observed. Frontal activity may reflect an executive function which allocates resources and/or organizes the processing stages of the task..$^{15,21}$ Some evidences also fit with neuropsychological data, showing that arithmetic deficits in adults, who developed high arithmetic skill prior to brain insult, tend to be associated with parietal brain lesions. ${ }^{16,17}$ In summary, our data show that skill acquisition for real-time arithmetic recognition task in learning disabilities children who had brain training, revealed the electroencephalographic activity changes especially the alpha and beta frequency bands. Skills acquisitions were reflected in systematic electroencephalographic activity changes. However, further empirical studies are needed to evaluate the suggested frequency bands of electroencephalographicrelated to realtime arithmetic recognition task.

\section{CONCLUSION}

Practicing the real-time arithmetic recognition task might significantly increase both alpha and betafrequency bands in learning disabilities children. The brain training might improve cognitive performance in learning disabilities children indexed by the electro-encephalographic activity.

\section{ACKNOWLEDGEMENT}

We thank all of the participants who involved in this study as main data provider.

\section{REFRENCES}

1. Logan GD. Towards an instance theory of automatization. Psychol Rev 1988; 95:492-527.

2. Newell A and Rosenbloom SP. Mechanisms of skill acquisition and the law of practice, In. JRAnderson (ed.), Cognitive Skills and their Acquisition, Erlbaum, Hillsdale, N.J., 1981.

3. Rickard TC. Bending the power law: a quantitative model of the transition from algorithm to association, Dissertation Proposal, University of Colorado, Boulder, CO., unpublished.

4. Bailey SP, Hall EE, Folger SE and Miller PC. Changes in EEG during graded exercise on a recumbent cycleergometer. J Sports Sci Med 2008; 7:505511.

5. Gutmann B, Mierau A, Hulsdunker T, Hildebrand C, Przyklenk A, Hollmann W and Struder HK. Effects of Physical Exercise on Individual Resting State EEG Alpha Peak Frequency. Neural Plast 2015; 717312.

6. Moraes $H$, Deslandes $A$, Silveira $H$, Ribeiro $P$, Cagy $M$, Piedade R, et al. The effect of acute effort on EEG in healthy young and elderly subjects. Eur J Appl Physiol 2011; 111(1): 67-75. 
7. Allen JJ, Coan JA and Nazarian M. Issues and assumptions on the road from raw signals to metrics of frontal EEG asymmetry in emotion. Biol Psychol 2004; 67:183-218.

8. Crabbe JB and Dishman RK. Brain electrocortical activity during and afterexercise: A quantitive synthesis. Psychophysiol 2004; 41:563-574.

9. Inouye $\mathrm{T}$, Shinosaki $\mathrm{K}$, Iyama A and Matsumoto $\mathrm{Y}$. Localization of activated areas and directional EEG patterns duringmental arithmetic. Electroencephalogr Clin Neurophysiol 1993; 86:224-230.

10. Chwilla DJ and Brunia CHM. Effects of emotion on eventrelated potentials in an arithmetic tasks. J Psychophysiol 1992; 6:321-332.

11. Roster F, Schumacher $G$ and Sojka B. What the brain reveals when it thinks: Event-related potentials duringmental rotation and mental arithmetic. German J Psychol 1990; 185-203.

12. Birbaumer $\mathrm{N}$, Elbert $\mathrm{T}$, Lutzenberger $\mathrm{W}$ and Rockstroh $\mathrm{B}$. EEG and slow cortical potentials in anticipation of mental tasks with different hemispheric involvement. Biol Psychol 1981; 13: 251-260.

13. Rosler $\mathrm{F}$ and Heil M. Toward a functional categorization of slow waves: Taking into account past and future events. Psychophysiol 1991; 28:344-364.
14. Ruchkin DS, Johnson R, Mahaffey D and Sutton S. Toward a functional categorization of slow waves. Psychophysiol1988; 25:339-353.

15. Ruchkin DS, Johnson R, Canoune H and Ritter W. Eventrelated potentials during arithmetic and mental rotation. Electroencephalogr Clin Neurophysiol 1991; 79:473-487.

16. Kolb B and Whishaw IQ. Funcdamental of Human Neuropsychology, $3^{\text {rd }}$ ed., Freeman, New York, 1990.

17. Levin HS and Spiers PA. Acalculia. In K.M. Heilman and E. Valenstein (eds.), Clinical Neuropsychology, Oxford University Press, New York, 1985.

18. Libert B, Gleason CA, Wright EW and Pearl DK.Time of conscious intention to act in relation to onset of cerebralactivity (readiness potential). Brain 1983; 106:623-642.

19. Luria A.The frontal lobes and the regulation of behavior. In K. Pribram and A. Luria (Eds.), Psychophysiology of the Frontal Lobes, Academic Press, New York, 1973.

20. Moscovitch M and Winocur G. The neuropsychology of memory and aging. In F.I. Craik and T.A. Salthouse (eds.), The Handbook of Aging and Cognition, Erlbaum, Hillsdale, N.J., 1992; 315-371.

21. Roland PE and Froberg L. Localization of cortical areas activated by thinking. J Neurophysiol 1985; 53:1219-1243.

\section{Authors Contribution:}

PS-Concept and design of the study, manuscript preparation, statistically analyzed and interpreted, critical revision of the manuscript; SCC-supported experimental device, helped in preparing first draft of manuscript.

Work attributed to: Brain Science and Engineering Innovation Research Unit, School of Anti-Aging and Regenerative Medicine, Mae Fah Luang University, Bangkok 10110, Thailand.

Orcid ID:

Dr. Phakkharawat Sittiprapaporn - (D) https://orcid.org/0000-0002-4103-9396

Shao-Chin Chang - (1) https://orcid.org/0000-0002-8764-2932

Source of Support: Grant No. 02201A601814 from Mae Fah Luang University, Thailand, Conflict of Interest: None. 